\title{
ESTRATEGIA DE MEJORAMIENTO Y CONSERVACIÓN DE LOS RECURSOS GENÉTICOS FORESTALES DE CHILE PARA ENFRENTAR EL CAMBIO CLIMÁTICO
}

\author{
Roberto Ipinzaํ, María Paz Molina², Braulio Gutiérrez³ y Oriana Ortíz ${ }^{4}$
}

\section{RESUMEN}

La mayoría de los conceptos convencionales sobre la conservación de la biodiversidad, en general, y de la diversidad genética en particular, están construidos sobre la idea errada de que la máxima aptitud de adaptación ya ha sido obtenida en la naturaleza. Muchos silvicultores y conservacionistas tienden a creer que la evolución asociada a la regeneración natural de las especies forestales siempre maximiza su aptitud reproductiva, y que mientras menor sea la intervención humana, mejor es la estrategia de conservación de los recursos genéticos. Sin embargo, el resultado de la selección natural refleja solo los efectos de las condiciones pasadas y no los efectos actuales como el cambio climático, las plagas, enfermedades, incendios, sequias y otros eventos que ocasionan una importante merma del acervo genético. Los cambios producidos son mucho más rápidos que la posibilidad que tienen las especies para adaptarse en forma natural, por lo mismo, la erosión genética y la eventual extinción son un riesgo latente.

El objetivo del presente trabajo es proponer una Estrategia de Mejoramiento y Conservación de los Recursos Genéticos Forestales de Chile, para asegurar la supervivencia, la adaptación y la evolución continúa de una especie forestal por más de 20 generaciones, en un ambiente continuamente cambiante, para así salvaguardar su potencial de adaptación.

La estrategia se elabora considerando criterios dinámicos en base al Sistema de Mejora de Poblaciones Múltiples (MPBS: Multiple Population Breeding System). El concepto de MPBS combina en forma segura la conservación sostenible ex situ de los recursos genéticos forestales con la preparación para los cambios eco-climáticos y la eficiente mejora genética forestal. Esta última se valida mediante simulación estocástica, en base a la variación genética, a la probabilidad de retener alelos raros, y a la estructura de la población de mejoramiento.

Palabras clave: Mejoramiento genético, conservación, cambio climático, estrategia, sistema de mejora de poblaciones múltiples.

\footnotetext{
1 Dr. Ingeniero de Montes. Instituto Forestal, Sede Valdivia. Chile. roberto.ipinza@infor.cl 2 Ingeniero Forestal Dr. (c). Instituto Forestal, Sede Biobío. Chile.mmolina@infor.cl 3 Ingeniero Forestal. Instituto Forestal, Sede Biobío. Chile. braulio.gutierrez@infor.cl 4 Oriana Ortiz. Ingeniera Forestal. Instituto Forestal. Sede Biobio Chile. oortiz@infor.cl.
} 


\section{BREEDING AND CONSERVATION STRATEGY FOR THE CHILEAN FOREST GENETIC RESOURCES, FACING THE CLIMATE CHANGE}

\section{SUMMARY}

Most of the conventional concepts about biodiversity conservation, particularly those about genetic diversity, have been developed on the missed idea that the maximum adaptation aptitude has already been obtained in the nature. Many foresters and conservationists believe that the evolution and natural regeneration always maximizes the reproductive aptitude of forest species, so as smaller human intervention, as better is the genetic resources conservation strategy. Anyway, the result of the natural selection shows the effects of past conditions, but not present effects (like climatic change, plagues, diseases, fires, droughts). These last effects could bring an important decreasing of the genetic pool. The environmental changes are faster than the natural adaptive species reaction, then, the genetic erosion and the possible extinction are latent risks.

The objective of this paper is to propose a National Strategy of Conservation for the Chilean Forest Genetic Resources, to assure the survival, the adaptation, and the forest species continue evolution for more than 20 generations under a continuously changing environment, aimed to safeguard theirs adaptation potential.

The strategy is elaborated over the basis of the Multiple Population Breeding System (MPBS). The MPBS concept combines in a safe form the sustainable ex situ conservation of the forest genetic resources with their adaptation for the eco-climatic changes. Besides this, it considers the efficient forest genetic improvement too. The forest breeding is validated by stochastic simulation, on the basis of the genetic variation, the probability of retaining rare alleles, and the structure of the improvement population.

Keywords: Breeding; conservation; climatic change; strategy; multiple populations breeding system. 


\section{INTRODUCCIÓN}

El Plan de Acción Chile 2030 (LCSAR-MINAGRI-FIA, 2011), elaborado por el Banco Mundial, identifica al mejoramiento genético como un pilar fundamental para mejorar la competitividad del país. En tal contexto, la protección y conservación de los $\mathrm{RGF}^{5}$, es la única alternativa que posibilita que el mejoramiento genético cumpla el papel identificado por el Banco Mundial.

Chile no cuenta con un programa nacional específico para la conservación de los RGF, sin embargo existen iniciativas que contribuyen a este logro. La principal de ellas corresponde al SNASPE ${ }^{6}$, donde se ha verificado un aumento del número de unidades, desde 87 en el año 1994 hasta 96 en la actualidad. El sistema comprende 32 parques nacionales, 49 reservas nacionales y 15 monumentos naturales, los que en su conjunto representan una superficie de 14,34 millones de hectáreas, equivalentes al 19\% del territorio nacional. Dentro de esta superficie, sólo 4 millones de hectáreas corresponden a bosques, lo que representa un $29 \%$ de las 13,5 millones de hectáreas (CONAFCONAMA-BIRF, 1999) que componen los bosques naturales de Chile. Por esta razón, la conservación en Chile normalmente se ha considerado de naturaleza in situ, donde los parques y las reservas forestales son las figuras primordiales.

Por otra parte, la superficie cubierta por $\mathrm{APP}^{7}$ ha crecido en forma importante en Chile a partir de los años 90 . El primer catastro realizado en 1997 por CIPMA $^{8}$, registraba 39 APP que cubrían cerca de 400 mil hectáreas. Actualmente, se estima la existencia de unas 500 APP que cubren más de 1,4 millones de hectáreas. Sin embargo, el país no cuenta con una definición operacional que permita identificar cuáles APP cumplen con criterios mínimos para ser consideradas como parte de un Sistema Nacional de Conservación. Tampoco dispone de un marco legal que reconozca a las APP existentes o promueva su creación en sitios prioritarios (Parques para Chile, 2011).

Tanto el SNASPE como las APP contribuyen, mediante conservación in situ, a perpetuar y proteger la integridad del acervo genético ${ }^{9}$ natural y los procesos evolutivos que afectan a dicho acervo, incluyendo aquellos provocados por el cambio climático.

Estudios llevados a cabo bajo condiciones ambientales uniformes han encontrado una marcada diferenciación genética en muchas características cuantitativas entre poblaciones de una misma especie provenientes de regiones específicas (Cornelius et al. 1996; Boshier y Henson 1997; Navarro et al. 2002). Esto significa que, si bien puede existir el potencial de adaptación ${ }^{10}$ dentro del acervo genético de una especie, debido a

\footnotetext{
5 RGF: Recursos Genéticos Forestales. Valor económico, científico o social de los materiales hereditarios contenidos dentro de las especies y entre ellas. http://www.fao.org/DOCREP/006/T0743S/T0743S00.htm\#glo; El material genético de valor real o potencial. Convenio sobre la Diversidad Biológica. Artículo 2. PNUD 1992 6 SNASPE: Sistema Nacional de Áreas Protegidas del Estado

7 APP: Áreas Protegidas Privadas.

8 CIPMA: Centro de Investigación y Planificación del Medio Ambiente.

9 Acervo genético = la suma total de toda la variación genética en la población de mejoramiento de una especie y especies estrechamente relacionada capaces de cruzarse con ella.

10 Adaptación: Proceso evolutivo por el cual los organismos llegan a estar mejor capacitados para vivir y reproducirse en variadas condiciones ambientales.
} 
que ya que existe una adecuada diversidad genética ${ }^{11}$ en esta, es probable que algunos de sus individuos no lo tengan. Es decir, los alelos necesarios para la adaptación al cambio climático en una región específica pueden estar presentes solamente, o en frecuencias mucho más altas, en poblaciones de otras regiones

De acuerdo a Koskela et al. (2007), la diversidad genética es la forma a través del cual los árboles pueden sobrevivir, adaptarse y evolucionar bajo las condiciones de cambios ambientales. La diversidad genética también es necesaria para mantener la vitalidad del bosque y protegerlo de plagas y enfermedades forestales. Además la diversidad genética también cumple un papel crucial en mantener la biodiversidad biológica forestal, tanto a nivel de especies como del ecosistema.

Ipinza (2000) indica que el hombre está obligado a intervenir, en forma positiva los bosques naturales, en especial si este ha jugado un papel en el deterioro de los recursos genéticos, a través de la selección disgénica ${ }^{12}$. No obstante, la actual variación genética es clave para enfrentar un futuro incierto derivado del cambio climático.

Kremer (2007) ha sugerido que el cambio climático, como inductor de evolución, es un fenómeno que ya se está produciendo y que los árboles han comenzado a adaptarse a un aumento en la concentración de dióxido de carbono atmosférico. Dicho autor establece que la adaptación de los árboles del bosque puede producirse en pocas generaciones, o en menos de 200 años.

En este contexto, Kremer (2007) sugiere que la transferencia de material reproductivo efectuada por el hombre, puede ser necesaria para mejorar la adaptación local de especies raras y escasas. De la misma forma Mátyás (2007) considera que la intervención humana es necesaria para facilitar la adaptación de los árboles del bosque al cambio climático y recomienda desarrollar una guía flexible para usar material reproductivo, e incorporar el cambio climático y la adaptación a los Programas Nacionales de Bosques de los países europeos. Por su parte, Lefèvre (2007) analiza la estructura organizacional para la conservación de los recursos genéticos en Francia y estima los efectos del cambio climático sobre la estrategia de conservación de genes, estableciendo que es necesario reevaluar y mejorar las redes de conservación de genes en el contexto del cambio climático, ya que estas deben ampliarse hacia la plasticidad ${ }^{13}$, adaptación y migración ${ }^{14}$ potencial de las especies forestales.

Se debe tener en cuenta que la actual estructura administrativa del Estado, organizada en regiones, es de vital importancia para una adecuada planificación

11 Diversidad genética, concepto general: Cantidad de variabilidad genotípica en una población; Definición cuantitativa: número de diferentes alelos por locus y la proporción de loci con más de un alelo en una especie o población.

12 Selección disgénica: Proceso que es perjudicial a la calidad genética de una población. Usualmente aplicado a las acciones humanas, tales como el floreo, que puede reducir el acervo genético local de una población natural.

13 Plasticidad: Capacidad de una especie de ocupar una mayor amplitud ecológica, presentando distintas respuestas que le permiten sobrevivir en cada medio ambiente diferente, sin que se produzcan cambio genéticos en ella.

14 Migración: La migración o flujo génico, ocurre cuando los individuos se trasladan de una población a otra y se cruzan con la segunda. 
estratégica de la conservación de los recursos genéticos forestales (RGF) de Chile. Finalmente, es necesario considerar a las Universidades, Centros de Investigación y al sector privado, propietario de bosques nativos, que mediante incentivos y acuerdos vinculantes pueden jugar un importante papel en la conservación de los RGF a través de la Red de Conservación de Recursos Genéticos Forestales de Chile.

\section{SISTEMA DE MEJORA DE POBLACIONES MÚLTIPLES}

\section{Conservación In Situ y Ex Situ}

La conservación, entendida como una disciplina dedicada a la protección, rescate, mantención, estudio y uso sustentable del patrimonio biológico de un país, es vital para mantener la diversidad genética de especies de este o de una región, así como sus interacciones y los procesos evolutivos que las originan. La conservación de los RGF se puede practicar bajo dos modalidades; in situ, es decir en el lugar donde crecen en estado natural o silvestre, o ex situ, fuera del lugar donde crecen en estado natural, como es el caso de los distintos bancos de germoplasma y rodales plantados de conservación (Salazar et al., 2006). Ambas opciones son complementarias para la conservación de especies y de su variación genética intraespecífica. En ocasiones los conceptos de ex situ e in situ se confunden, debido a que en conservación agronómica se suele usar el concepto ex situ sólo para bancos (de semillas, polen u otros), sin considerar a los rodales de conservación plantados en campo; por otra parte asocian los cultivos en campo como una forma de conservación in situ (Eriksson et al., 2006).

La conservación in situ de los recursos genéticos, es decir bajo las condiciones propias de su hábitat natural, ya sea en bosques productivos o en áreas protegidas, permite la interacción con otras especies y organismos a largo plazo, dejándolas evolucionar en su medio natural, en conexión con la evolución de las condiciones ambientales de su localidad. El término se aplica a la regeneración natural que permite preservar el potencial de adaptación de las especies, pues hace posible que la selección y el flujo genético actúen como procesos que caracterizan la evolución.

Un sistema de conservación de RGF que permita hacer mejoramiento y conservación ex situ requiere identificar a la población de mejoramiento ${ }^{15}$ como la estructura clave del sistema. Esto, por sí sólo no es suficiente para hacer que este sistema funcione y evolucione, sino que requiere además de una estrategia para que este trabaje en forma ordenada y eficiente. En la Figura $\mathrm{N}^{\circ} 1$ se ilustra la pirámide de Burdon ${ }^{16}$, donde se conceptualiza el mejoramiento y la conservación de los RGF de Chile. En la base de la pirámide, en sentido horizontal, se representa la diversidad genética, mientras que en su eje vertical, a medida que se estrecha la variación, se aumenta la ganancia genética ${ }^{17}$.

15 Población de mejoramiento: Un grupo de individuos seleccionados desde una población para ser utilizados en un programa de mejoramiento. Inicialmente la selección es fenotípica. En especie con un rango amplio, hay varias o muchas poblaciones de mejora mas o menos separada, cada una está designada para proveer progenies adecuadas a una región geográfica particular.

16 Presentación dada en el congreso "Western Forest Genetics Association meeting, 28 August 1995, Victoria British Columbia.

17 Ganancia Genética: Cambio genotípico originado por la selección artificial en un rasgo específico. La ganancia se expresa en términos de cambios por generación o cambios por año. La ganancia esta determinada por la 
En este esquema se observa que la amplia base (población de RGF) está conformada por bosques naturales, representados mayoritariamente en el SNASPE; luego la población de mejoramiento, constituida fundamentalmente por pino y eucalipto de las empresas forestales y ensayos de progenie y procedencia e introducción de especies conformada por INFOR y otros; la cúspide de la pirámide esta conformada por las poblaciones de producción, fundamentalmente de pino y eucalipto y algunas otras especies, donde se concentra la mayor ganancia genética generada por sus respetivos programas de mejora.

Los largos periodos de madurez de la inversión en mejora y conservación genética ex situ, sumados a los normalmente escasos recursos financieros destinados a este fin, sugieren la necesidad de complementar los esfuerzos técnicos y los recursos financieros, de modo de propender a un enfoque de cooperación entre los actores vinculados a estas materias. En Chile, existen distintas organizaciones que trabajan en mejoramiento y conservación de recursos genéticos, tanto privadas como públicas, pero no existe una coordinación para optimizar su gestión, papel que está actualmente asumiendo el Ministerio de Agricultura a través del $\mathrm{FIA}^{18}$, de acuerdo a las recomendaciones del Banco Mundial.

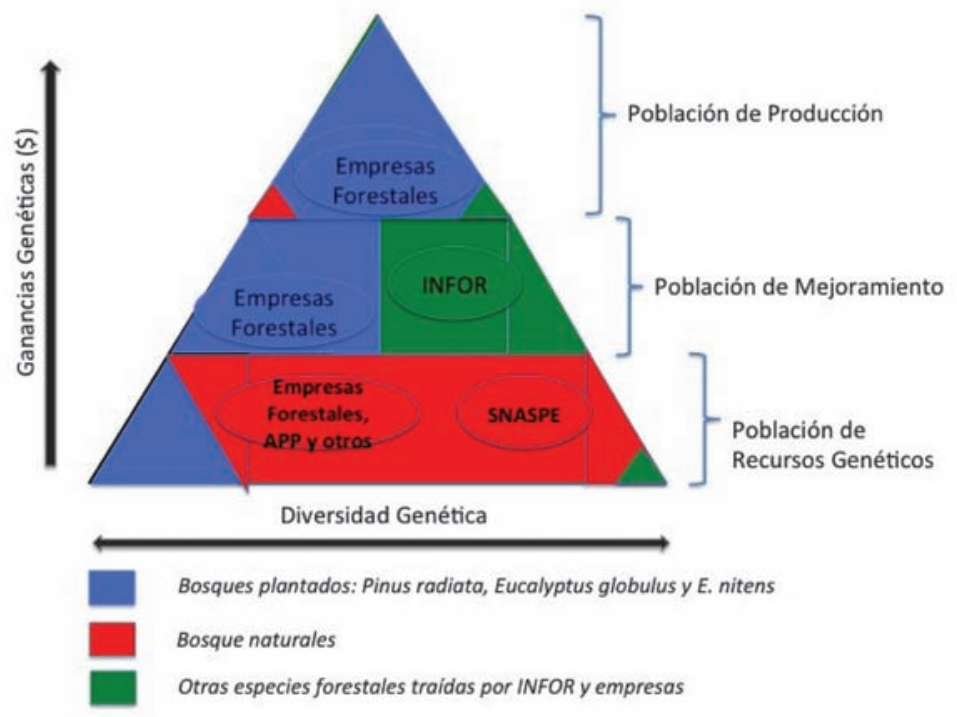

Figura $N^{\circ} 1$.

ESQUEMA DE LA PIRÁMIDE DE BURDON, APLICADO A LOS RECURSOS GENÉTICOS FORESTALES DE CHILE.

El desarrollo de una estrategia de conservación y mejoramiento de RGF considera

intensidad de selección, la variación de los progenitores, y la heredabilidad de un rasgo dado.

18 FIA: Fundación para la Innovación Agraria del Ministerio de Agricultura de Chile. 
dos etapas iterativas. Primero, se define el sistema de mejoramiento y su sistema silvícola asociado. En segundo lugar, se definen y evalúan las opciones de ganancia y variabilidad que se pueden generar. Los resultados de la evaluación deben reflejar la estructura del sistema, y de su funcionamiento se generan y evalúan nuevas opciones. El proceso iterativo es ampliable, y el índice de la iteración debe ser ajustado para que se pueda mantener un equilibrio entre el aumento de las ganancias genéticas en el corto plazo y la variabilidad genética en el largo plazo. En este sentido un límite adecuado es de 20 generaciones.

El objetivo primario de la conservación de recursos genéticos es asegurar la supervivencia, la adaptación, y la evolución continúa de una especie en un ambiente continuamente cambiante. Según Eriksson (2000), el objetivo clave de la conservación genética es salvaguardar el potencial de adaptación. Mientras dicha población este conservada ex situ o in situ, a largo plazo, y en forma estructurada, será posible que exista suficiente variación genética para enfrentar futuros cambios en el clima o en las prioridades de mejora. De esta forma, mediante muestreo genético de la población conservada, en el futuro el hombre podrá realizar progresos genéticos en la dirección que se requiera. Podrá por ejemplo, mejorar las características de una determinada especie de latifoliada que esté representada en un rodal de conservación, definiendo en este caso los objetivos específicos que le interese cumplir, como por ejemplo crecimiento, calidad de madera, u otros.

Es necesario insistir que para alcanzar estos objetivos en forma exitosa, se deben mantener rodales de conservación ${ }^{19}$ con una amplia variabilidad genética, capturada mediante muestreo genético realizado sobre las distintas poblaciones de los bosques actuales (primarios, secundarios, residuales) y mediante el manejo forestal crear las condiciones ex situ favorables para la adaptación rápida de cada especie (Müller-Using y Martin, 2011). Un requisito previo y general para la evolución es la regeneración natural o asistida de los RGF. Con esta última, se pueden aplicar medidas específicas en aquellas situaciones donde existen dificultades para que las especies se mantengan por si solas durante sucesivas generaciones.

La mayor parte de las estrategias de conservación estáticas se realizan en la modalidad ex situ, aunque también puede realizarse conservación ex situ dinámica. En este caso, se puede llevar a cabo su manejo de modo dinámico, bajo unas condiciones que pueden ser muy próximas a las del lugar de origen, haciendo una silvicultura cercana a la naturaleza. Cuando estos rodales de conservación se sitúan bajo climas diferentes se promueven adaptaciones e interacciones diferentes a las de su primitiva procedencia. Pese a su origen artificial, el objetivo es dejar a la población evolucionar y reproducirse de modo cercano a lo natural.

\section{Conservación de Recursos Genéticos (CRG): Dinámico y Estático}

Existen dos tipos de aproximaciones, según se considere o no el mantenimiento de

19 Rodales de conservación: Es un rodal designado para la conservación in situ o ex situ de los RGF de una especie prioritaria y que formara parte de la red. El rodal debe tener su propio plan independiente de ordenación, pero sincronizado con el plan de la red. 
los procesos genéticos como parte de la estrategia de conservación, en la Figura №2 se muestra el significado de manejar los recursos genéticos en forma dinámica y estática.

La conservación dinámica de recursos genéticos tiene por objetivo que la variación genética existente en el material conservado no sólo garantice su adaptabilidad actual, sino también la evolución continua de la especie. Trata de mantener una amplia diversidad genética y potencial adaptativo, mediante la doble influencia de la presión selectiva ${ }^{20}$ del medio y de la reproducción sexual. De este modo, van apareciendo nuevos genotipos por recombinación y apareamiento, sin llevarse a cabo una selección fenotípica de los árboles progenitores. Esta estrategia requiere una protección efectiva frente a la introgresión genética ${ }^{21}$ desde el exterior, así como frente a incendios $u$ otro tipo de amenazas sobre la población conservada. La conservación dinámica puede requerir considerable espacio y costos y se puede enfrentar al uso actual que recibe la población a proteger o el territorio colindante.

La conservación estática de recursos genéticos considera el principio de preservar la diversidad existente y está ligada a la creación de colecciones que mantienen composiciones genéticas específicas, sin respuesta a posibles cambios en el ambiente, y sin generar nueva diversidad. Los métodos de conservación estática se aplican a un material geneticamente bien identificado y que estabiliza su composición genética actual, definida por el material incorporado a la colección o banco de genes.

Los esfuerzos se concentran en la conservación de genotipos individuales, que se pueden preservar de diversas formas, como semillas, polen, partes de plantas o plantas enteras. La conservación se puede realizar en bancos de semillas, cuya duración la define la pérdida de la capacidad germinativa de las mismas, o en bancos o colecciones clonales propagadas vegetativamente, 0 mantenidas indefinidamente mediante técnicas de crioconservación (almacenadas sin daño a temperaturas de $-196{ }^{\circ} \mathrm{C}$ en nitrógeno líquido). A largo plazo, su inconveniente se deriva de impedir los efectos de la recombinación derivada de la reproducción sexual y de la selección, que permiten aparecer una diversidad que responda a nuevas condiciones ambientales.

En Chile, en relación a este tipo de conservación ex situ, bajo la modalidad de bancos de germoplasma se conservan del orden de 600 especies y 67 mil accesiones $^{22}$. El $88 \%$ de las accesiones corresponden a plantas cultivadas de interés para la alimentación y la agricultura, y se representan mayoritariamente por semillas en los bancos del INIA. Respecto a las colecciones de RGF, la información de INIA-FAO (2008) permite establecer que estas involucran a cerca de 30 especies, con un total de 2.730 accesiones. De ellas la inmensa mayoría corresponde a las especies exóticas Pinus radiata y Eucalyptus spp.

20 Presión selectiva: Es aquella fuerza del medio ambiente: natural o artificial que es capaz de generar cambios en la frecuencia de alelos y por consiguiente en las características fenotípicas de la población.

21 Introgresión genética: Es el movimiento de genes de una población a otra a través de la hibridación seguida por el retrocruzamiento. Comúnmente se refiere al movimiento de genes desde una especie a otras o entre subespecies que están aisladas geográficamente.

22 Accesiones: Se denomina así a la muestra viva de una planta, cepa o población mantenida en un banco de germoplasma para su conservación y/o uso. Una especie puede estar representada por varias entradas que se diferencian por el tipo de población al que pertenecer (i.e. variedad primitiva, variedad tradicional, variedad mejorada, líneas avanzadas de mejoramiento, plantas silvestres) y/o por su origen (lugar de recolección o creación). 
(70\%, de las accesiones); un 19\% (517 accesiones) corresponden a la especie nativa Nothofagus alpina; y el $11 \%$ restante (312 accesiones) se distribuye irregularmente en cerca de 25 especies forestales nativas y exóticas.

En consecuencia, las colecciones forestales conservadas ex situ en bancos de germoplasma, corresponden a pocas especies, almacenadas en bancos de pocas instituciones y donde la enorme diversidad de flora del bosque nativo se encuentra marginalmente representada. Por otra parte, en general, la seguridad de las colecciones de RGF almacenadas en el país es baja, INIA-FAO (2008) indican que solo un 10\% están como semillas en bancos base ${ }^{23}$, el resto se encuentra en bancos activos ${ }^{24} y$ fundamentalmente en colecciones de trabajo.

El documento también indica que existe un escaso nivel de documentación de las colecciones y que muchas se han establecido en el marco de proyectos de investigación con un horizonte de término limitado, por lo tanto no existe seguridad de su permanencia en el largo plazo. En consecuencia, un muy bajo porcentaje de las accesiones está almacenado en forma segura, de largo plazo y con adecuada documentación.

Otras colecciones de conservación ex situ son los jardines botánicos, los que también se presentan en un número muy limitado en el país, siendo los Jardines Botánicos de Viña del Mar (CONAF) y de Valdivia (UACH) los más importantes (EMBRAPA, 2006).

23 Banco base: Es una colección que se mantiene para las generaciones futuras; conservación a largo plazo; mínimo 100 años. Para dicha conservación se utilizará una humedad entre el 3 y el 7\% (a menor humedad, mayor viabilidad a largo plazo), y una temperatura de $-20^{\circ} \mathrm{C}$. Esta colección es inaccesible a no ser que sea estrictamente necesario.

24 Banco activo: Es una colección que se usa para realizar los estudios científicos. Es accesible también para donaciones. Se mantiene en condiciones de conservación a medio plazo (unos 10 años): humedad entre el 3 y el $7 \%$ y en frigorífico (entre 0 y $5^{\circ} \mathrm{C}$ ). 

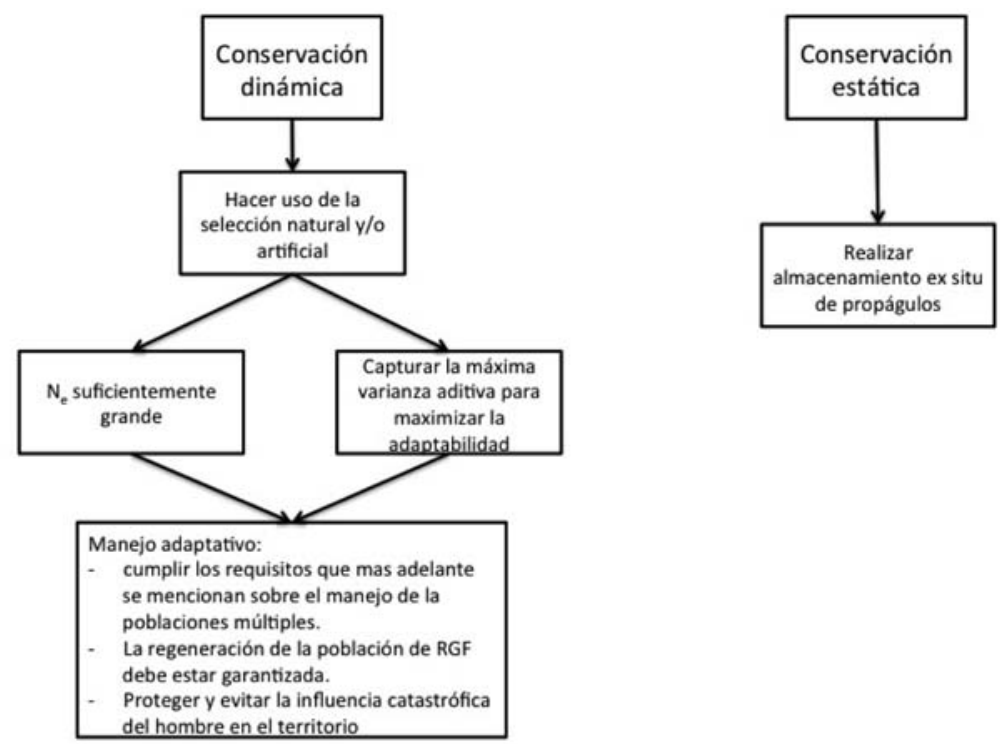

Figura $\mathrm{N}^{\circ} 2$.

MODELO DE CONSERVACIÓN DE RECURSOS GENÉTICOS: DINÁMICO Y ESTÁTICO.

\section{Modelo MPBS}

Los Parques Nacionales y las Reservas Forestales (SNASPE) constituyen el sistema convencional de conservación in situ más usado en el mundo. Dada la velocidad del cambio climático y la relativamente lenta respuesta adaptativa de los bosques, este enfoque tradicional se debe complementar con otro, como el sistema de mejora de poblaciones múltiples (MPBS). El concepto de MPBS combina seguridad, la conservación sostenible y ex situ de los recursos genéticos forestales, y la preparación para los cambios eco-climáticos, con una amplia varianza genética ${ }^{25}$ para hacer mejoramiento genético forestal en el futuro. El esquema de MPBS ya ha sido aplicado en algunos países (Pliûra y Eriksson 1997).

El Sistemas de Mejora de Poblaciones Múltiples fue originalmente desarrollado para el mejoramiento genético por Namkoong (1976) y posteriormente adaptado a la conservación de recursos genéticos (Namkoong 1984), constituyendo hoy un sistema fundamental para la conservación de RGF.

Según el MPBS, la población de mejora de recursos genéticos puede consistir de un conjunto de subpoblaciones pequeñas (Figura $N^{\circ} 3$ ), cada una con un tamaño poblacional efectivo ${ }^{26}$, o número de entidades genéticas no emparentadas, determinado en función de la proporción de genes que se pretende conservar. La subpoblaciones

25 Varianza genética: Es la varianza fenotípica resultante de la presencia de distintos genotipos en la población. 26 Tamaño efectivo ( $\mathrm{Ne}$ ): Se refiere a individuos no emparentados. 
representan distintos criterios de selección, fundamentalmente rasgos adaptativos, por ejemplo, crecimiento juvenil en altura, características fenológicas, resistencia a factores adversos (enfermedades, heladas, sequía), entre otras. Las sublíneas son definidas como replicas de la población de mejora, donde cada una representa a una región de procedencia. Así, considerando la estructura de colaboración publico privada para desarrollar el programa de mejora y conservación ex situ, es altamente probable que las regiones de procedencias (Vergara, 2000) localizadas en diferentes condiciones ecológicas, debido a la presión de selección que ejerce el cambio climático, tiendan rápidamente a diferenciarse, y asimilarse a un sistema de mejoramiento de poblaciones múltiples. La estimación de la plasticidad fenotípica ${ }^{27}$ de los distintos rasgos permitirá evaluar la posibilidad de adaptación al cambio climático (Pliûra, 2004).
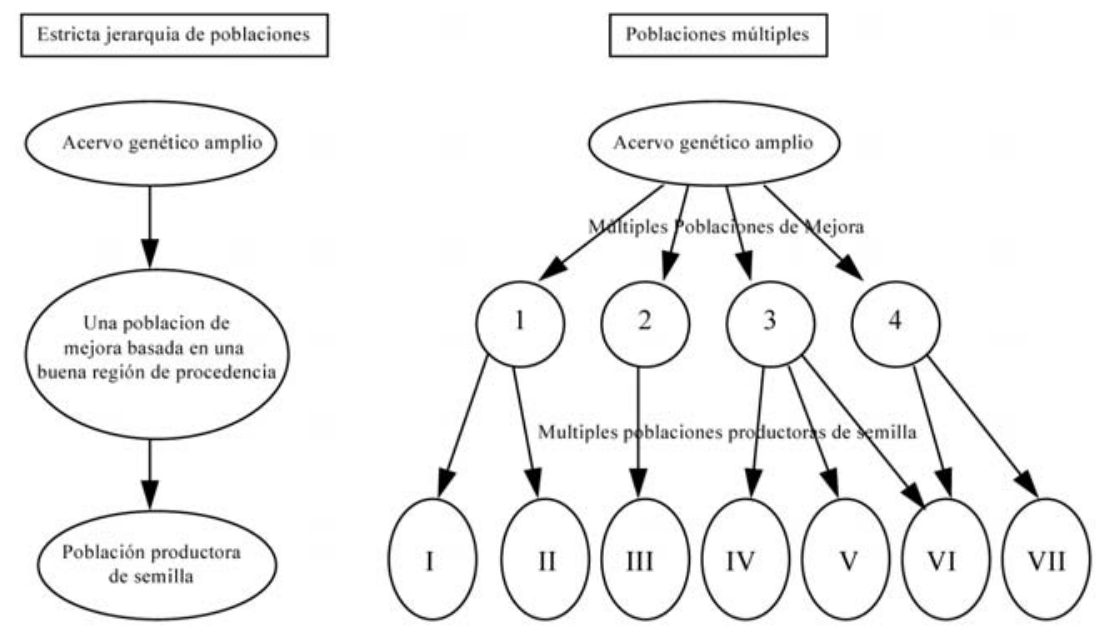

Figura $\mathrm{N}^{\circ} 3$.

\section{COMPARACION ENTRE MODELO DE POBLACIONES MÚLTIPLES Y ESQUEMA JERARQUIZADO DE POBLACIONES}

\section{Selección y MPBS}

Para capturar los alelos de frecuencias superior a 0,01, con 0,99 de probabilidad, la población de conservación genética deberá estar constituida por alrededor de 750 - 1.150 individuos (Gregorius, 1980). Por lo tanto proporcionarán suficiente diversidad genética para la conservación genética sostenible de largo plazo y para los propósitos de mejoramiento genético también de largo plazo.

Ahora, si se considera capturar los alelos de más baja frecuencia, el número de individuos a incluir en la población de conservación puede aumentar rápidamente. Debido a los riesgos del cambio climático y de desastres naturales o antropogénicos, se

27 Plasticidad fenotípica: $\mathrm{PF}=\left(\mathrm{V}_{\mathrm{G} \times \mathrm{A}}+\mathrm{V}_{\mathrm{A}}\right) /\left(\mathrm{V}_{\mathrm{GXA}}+\mathrm{V}_{\mathrm{A}}+\mathrm{V}_{\mathrm{G}}\right)$, donde: $\mathrm{V}_{\mathrm{G} \times \mathrm{A}}=$ Varianza asociada con la interacción genotipo ambiente; $\mathrm{V}_{\mathrm{A}}=$ Varianza ambiental, $\mathrm{y} \mathrm{V}_{\mathrm{G}}=$ Varianza genética . 
requerirán poblaciones de conservación genética con más de mil árboles. Un número relativamente pequeño de genotipos en las sub poblaciones ayudará a la fijación de los nuevos genes originados de las mutaciones y puede acelerar la evolución.

Una condición previa para hacer operativa la selección natural o artificial, es que la población de recursos genéticos sea lo bastante grande para capturar la alta variación genética aditiva ${ }^{28}$ y así evitar la deriva genética ${ }^{29}$. De acuerdo al concepto de MPBS, la población de mejoramiento y conservación de recursos genéticos de una especie determinada debe consistir de aproximadamente 10 a 20 subpoblaciones, cada una de ellas con un tamaño efectivo $\left(\mathrm{N}_{\mathrm{e}}\right)$ de 50 individuos, los que totalizan una población de 5001000 individuos (Gregorius, 1980). Lo anterior, basado en la probabilidad de guardar genes con frecuencias superiores a 0,01 , y evitar una severa endogamia ${ }^{30}$ en las subpoblaciones (Pliura y Eriksson 1997). Con un tamaño efectivo de 50 individuos por subpoblación, la tasa de endogamia será el 1\% por generación, ya que el coeficiente de endogamia $F=1 /$ $\left(2 \mathrm{~N}_{\mathrm{e}}\right)=0,01$ (Varela y Eriksson, 1995), es reconocido como satisfactoriamente bajo (Danell, 1993). Esto también corresponde a la tasa de pérdida de variación aditiva (Eriksson, 2000), ya que el aumento del coeficiente de endogamia ${ }^{31}$ deriva de la misma fórmula $(F=0,01)$, asumiendo cruzamientos aleatorios.

Mediante un análisis teórico, Namkoong (1976) demostró que la división de la población de mejoramiento en varias subpoblaciones, es más eficiente que sólo una población de mejoramiento. También demostró que la hibridación de individuos de diferentes subpoblaciones produce un aumento de la varianza aditiva en la generación F2.

Desde el punto de vista del mejoramiento genético, particularmente en el manejo de las poblaciones élite ${ }^{32}$, la confección de líneas de mejoramiento, donde cada una de ellas puede estar formada por las 10 mejores familias, no emparentadas entre líneas, con alelos de frecuencias hasta 0,25 , sería suficiente para garantizar diversidad y sostenibilidad de los nuevos rodales, y proporcionar alto niveles de ganancias genéticas.

Los cambios en la diversidad genética de las poblaciones se representan por la endogamia promedio y por las estimaciones del tamaño efectivo de la población. El coeficiente de endogamia describe la proporción de locus homocigóticos en exceso, respecto de una población de cruzamientos aleatorios e infinitamente grandes. El coeficiente de endogamia para cada árbol en la población es determinado por el pedigrí de los árboles progenitores que permite determinar el grado de ancestros comunes. El cálculo es simple en pequeños pedigríes, pero se torna difícil en pedigríes complejos. En esta estrategia la endogamia se confina dentro de la subpoblación o sublínea, pero nunca entre sublíneas.

\footnotetext{
28 Variación genética aditiva: Representa la porción de la varianza total que es susceptible de ser transmitida de padres a hijos.

29 Deriva genética: Cambio en la frecuencia de genes o pérdida de genes en una población pequeña debido a efectos aleatorios. Comúnmente corresponde a una perdida de alelos raros.

30 Endogamia: Cruzamiento entre individuos emparentados. En especies de polinización abierta la endogamia provoca una pobre producción de semilla, baja germinación y una severa reducción del crecimiento.

31 Coeficiente de endogamia: Probabilidad que dos genes en un individuo sean idénticos por descendencia.

32 Población élite: Población probadamente superior mediante ensayos genéticos.
} 
Para capturar las adaptaciones que ya se han desarrollado en las poblaciones que conforman las distintas procedencias ${ }^{33}$ de una determinada especie, puede ser suficiente con un sistema de selección y muestreo que cubra los patrones geográficos de variación genética que la especie ha desarrollado en las particulares condiciones eco-climáticas de sus respectivas regiones de procedencia. Es importante considerar que si se realiza conservación ex situ, las procedencias también se deben mantener separadas para aprovechar su capacidad de adaptación.

La esencia de la conservación dinámica de los recursos genéticos al usar el concepto de MPBS es promover la adaptación, lo que se consigue al exponer la población de recursos genéticos a la selección natural y a los cambios climáticos. Por lo mismo, el material genético, ordenado por ejemplo en sublíneas o subpoblaciones, puede evolucionar en una variedad de direcciones (Figura $N^{\circ} 4$ ). Consecuentemente, una red de conservación de recursos genéticos ex situ debe establecerse en un amplio arreglo de condiciones eco-climáticas, bajo una colaboración público privada, donde la locación de las poblaciones es determinada por un mapa de futuros escenarios derivados del cambio climático.

El enfoque de poblaciones múltiples puede ser considerado tanto en las poblaciones de conservación in situ como en las ex situ. El desarrollo de la presente estrategia considera la conservación ex situ como un complemento de la in situ. La población de mejoramiento ex situ es colectada desde las regiones de procedencia (Vergara, 2000) localizadas en un amplio rango de ambientes, y luego expuestas a la selección natural y sometida a la evolución en una variedad de direcciones.

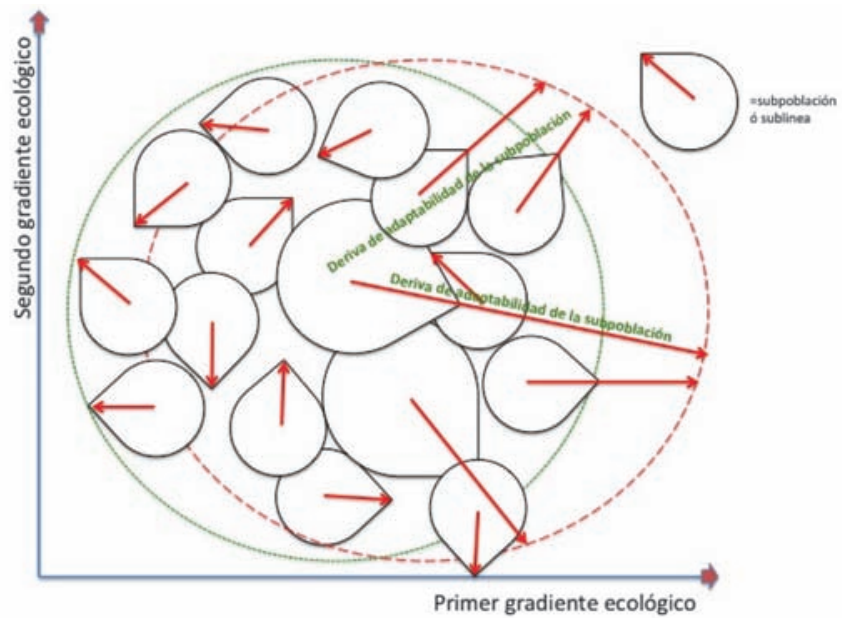

(Fuente: Pliûra, 1999)

\section{Figura $\mathrm{N}^{\circ} 4$}

\section{EVOLUCIÓN MULTIDIRECCIONAL DE SUBPOBLACIONES EN EL SISTEMA DE POBLACIONES MÚLTIPLES}

33 Procedencias: Fuente geográfica de la semilla, material vegetal o plantas procedentes de tal fuente. En general cabe afirmar que las especies forestales de distribución geográfica amplia presentan considerables variaciones en su anatomía, morfología y fisiología de acuerdo a esta. 
De ser necesario, las poblaciones de conservación de recursos genéticos pueden ser manejadas intensivamente para mejorar la adaptación de cada una de las subpoblaciones, para así aumentar la diferencia genéticas entre ellas, garantizar su sostenibilidad durante todos los períodos de la ontogénesis, asegurar la regeneración continua de la población de la especie objetivo, y protegerla contra todos los tipos de daño.

Para reducir al mínimo los costos, la conservación de recursos genéticos puede ser realizada en conjunto con la mejora genética y estudios genéticos específicos.

\section{Elementos claves para conservación de RGF en el modelo MPBS}

De acuerdo al horizonte de protección, las especies forestales pueden dividirse en aquellas para conservación en el corto - mediano plazo y otras para conservación en el largo plazo.

Para el largo plazo, mayor a 20 generaciones, es necesario considerar la dinámica de variación genética de una especie determinada, por ejemplo, en Argentina, la especie Austrocedrus chilensis presenta una mayor diversidad genética en poblaciones xéricas localizadas en la estepa patagónica, en propiedades privadas, fuera del sistema nacional de áreas naturales protegidas (Gallo et al., 2004). En este caso se debería proteger el borde o frontera sinuosa que delimita a la zona de estepa, y hacer además un muestreo para conservar ex situ la variabilidad. Sin menoscabo, que para usar la especie en un programa de mejoramiento en el corto - mediano plazo se seleccione árboles plus ${ }^{34}$ en los sitios donde mejor crezca la especie.

En el concepto del MPBS la conservación es dinámica, ya que las especies se benefician tanto de la selección natural como de la artificial y, de esta forma, pueden alcanzar altos niveles de adaptación a las cambiantes condiciones ambientales. También permite al mejorador responder a los posibles cambios de valor económico de los caracteres, producidos por ejemplo por plagas y enfermedades forestales.

En términos prácticos, el MPBS implica dividir la población de recursos genéticos en aproximadamente 20 subpoblaciones, que se mantienen físicamente en forma separada, en las que cada una debe tener un tamaño de población efectiva $\mathrm{N}_{\mathrm{e}}$, de aproximadamente 50 individuos. Ahora, si la población de mejoramiento tiene 20 subpoblaciones, el número census de árboles ${ }^{35}$ alcanzará a los 1000 . El dividir la población a conservar en varias subpoblaciones permite aumentar la varianza aditiva total con pérdidas marginales de la varianza dentro de la población (Eriksson et al., 1993). Luego, la endogamia estará en una tasa aceptable, debido a que con un tamaño de la población efectiva $\left(\mathrm{N}_{\mathrm{e}}\right)$ igual a 50 individuos, el coeficiente de endogamia $\left(F=1 / 2 N_{e}\right)$ alcanza apenas al 1\% (Varela y Eriksson, 1995).

34 Árbol plus: Individuo de características fenotípicas sobresalientes en algunos rasgos de interés para el programa.

35 Número census: Numero o tamaño actual de la población 
En la Figura $N^{\circ} 5$ se muestra que al aplicar el sistema de poblaciones múltiples, se logra la conservación dinámica de los recursos genéticos, promoviéndose la adaptación y la evolución de las especies.

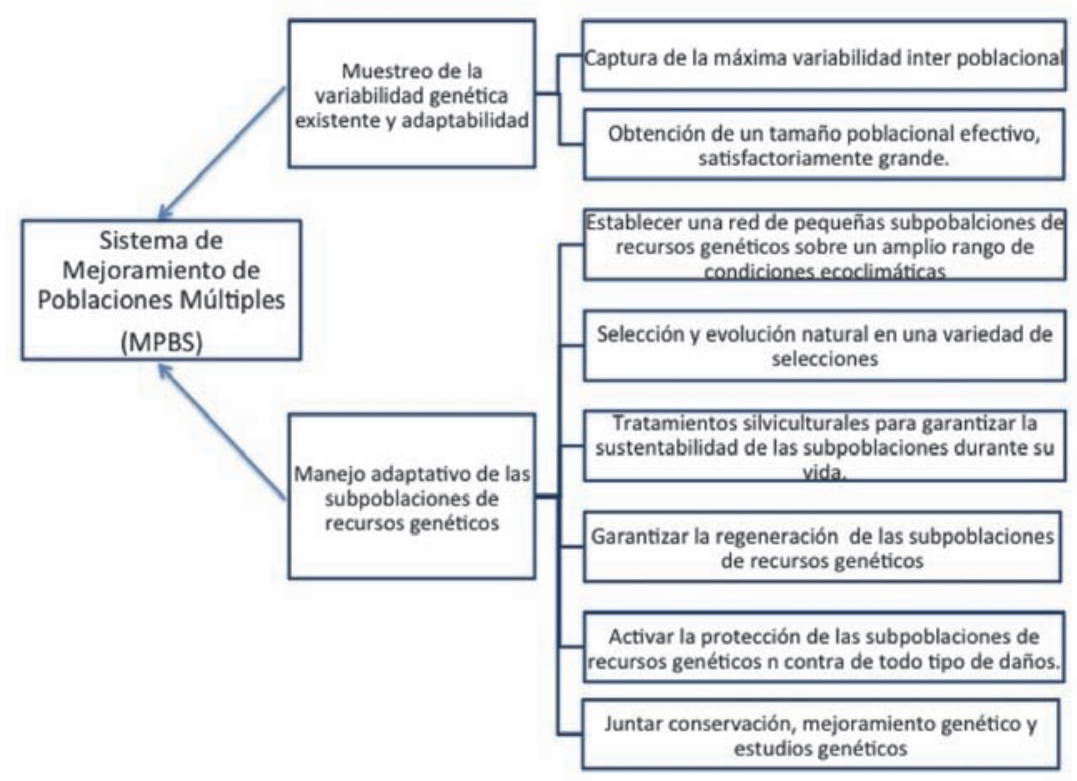

(Fuente: Pliûra, 1999).

\section{Figura $\mathrm{N}^{\circ} 5$}

\section{SISTEMA DE MEJORAMIENTO DE POBLACIONES MÚLTIPLES (MPBS): PROMUEVE LA ADAPTACIÓN Y EVOLUCIÓN DE LAS ESPECIES}

En la implementación del MPBS se pueden usar distintos grados de sofisticación, desde el manejo muy intensivo de la población de mejoramiento hasta la conservación in situ sin interferencia humana. La principal ventaja del MPBS es que combina la captura de la variabilidad genética total, existente en ese momento, dentro de cada subpoblación, y ello permite que las poblaciones de RGF se adapten a las condiciones ambientales. Otra ventaja es que la velocidad de la evolución puede ser más rápida en una población de 50 árboles que en una gran población de miles de árboles.

EI MPBS mas desarrollado involucra muestreo, plantación, manejo y regeneración de una población localizada en una amplia variedad de sitios. De esta forma cada una de las pequeñas subpoblaciones puede ser expuesta a varios tipos de condiciones climáticas y edáficas, y por lo tanto seguir diferente evolución. Varela y Eriksson (1995) establecen que la versión ex situ del MPBS es la más elaborada y que los ensayos de progenie y procedencia, que incluyen la variabilidad completa de una especie, pueden ser considerados como sus máximos exponentes. Por otro lado, si la especie es considerada 
en un programa de mejoramiento, debería ser de un costo muy bajo establecer la estructura MPBS ex situ con propósitos de conservación. La conservación ex situ se hace obligatoria para especies en vías de extinción o muy menoscabadas en su estructura genética. En estos casos es especialmente relevante incluir todos los alelos con frecuencias superiores al $1 \%$.

Dentro de la conservación in situ pueden existir distintos grados de variación, desde reservas forestales manejadas hasta aquellas reservas forestales no manejadas pertenecientes al Estado o a propietarios privados. En el primer caso, cuando se considera especies comunes y de amplia distribución, se sugiere el uso de MPBS in situ con una baja intensidad de manejo (Ferreira y Eriksson, 2006), el segundo caso sólo es pertinente si el objetivo es salvaguardar la estructura de especies endémicas. Otro nivel de conservación in situ de MPBS, de bajo costo, es considerar especies naturales que puedan coexistir en su rango de distribución.

Por último, y tal como lo reconoce la propia OCDE (2005 y 2011), muchos de los bosques nativos que no se encuentran en las áreas protegidas, continúan degradándose por incendios causados por el hombre y por la tala ilegal de especies valiosas, con el consecuente riesgo de pérdida de poblaciones locales y sus genes asociados. Desde el punto de vista del cambio climático, las especies que exhiben una amplia distribución geográfica, si bien no enfrentan un riesgo de sufrir procesos de extinción, sí pudieran verse afectadas poblaciones locales particularmente, las localizadas en los márgenes de su distribución (Hubert y Cottrell, 2007).

\section{Etapas para el Establecimiento de Sistema en Base de MPBS}

- Obtención de información base detallada, fundamentalmente mapas o shape en formato digital. Esta información se muestra en la Figura $N^{\circ} 6$ y se maneja con el GIS ${ }^{36}$, llamado DIVA ${ }^{37}$, aportado por LAFORGEN ${ }^{38}$

- Consulta a expertos: talleres de trabajo, encuesta Delphi, entre otros

- Definición de criterios de priorización (conservación, endemismo, productividad, rareza)

- Selección de especies prioritarias

- Determinación de la variación genética: Mediciones de la variabilidad genética y consideraciones de aislamiento geográfico.

- Evaluación del estado de conservación

- Identificación de poblaciones a conservar (preferentemente todas, cada una debería tener particularidades que aportan a la adaptación, tolerancia o productividad)

- Elección de las medidas de conservación adecuadas (en general deberían considerarse in situ y ex situ).

- Organización, planificación y ejecución de actividades

36 GIS: Geographical Information System

37 DIVA: Sistema geográfico de información, gratuito, para mapear y analizar datos geográficos. http://www.divagis.org/

38 LAFORGEN: Latin American Forest Genetic Resources Network. Es la red de RGF de América Latina y el Caribe 
- Preparación de guías de gestión para el manejo, evaluación y sustentabilidad en el tiempo del material genético resguardado.

- Establecimiento de un sistema de monitoreo que permita obtener información continua que facilite toma de decisiones o reingeniería de estrategias.

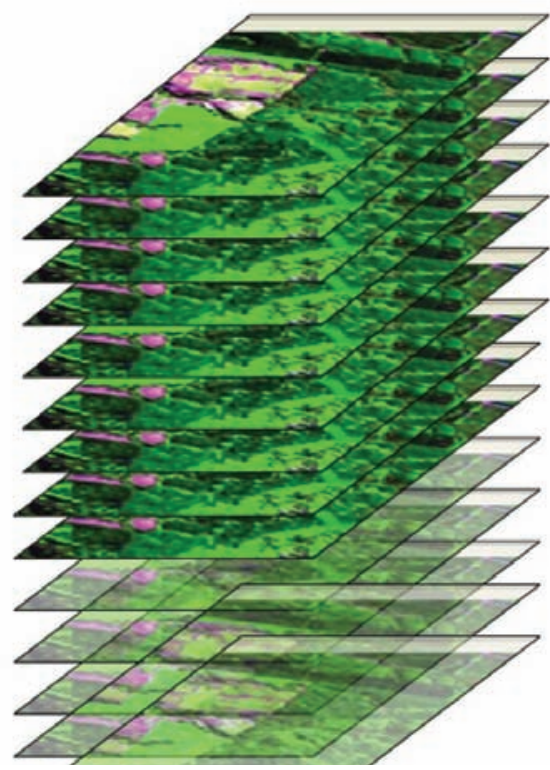

INVENTARIO INFOR

SNASPE

APP

CATASTRO

EMPRESAS FORESTALES

BANCOS ACTIVOS

BANCOS BASES

RODALES DE CONSERVACIÓN

POBLACIONES DE MEJORA

POBLACIONES DE PRODUCCION

BANCOS CLONALES

INVENTARIO ESPECIES RARAS

REGIONES DE PROCEDENCIAS

BASE I.G.M.

Figura $\mathrm{N}^{\circ} 6$

CAPAS DE INFORMACIÓN BASICA PARA ALIMENTAR EL GIS PARA LA CONSERVACIÓN Y MEJORAMIENTO DE LOS RGF DE CHILE

\section{ESTRATEGIA DE CONSERVACIÓN DE RGF EN CHILE: EL CASO DE ROBLE Y RAULÍ}

\section{Recursos en Bosques Naturales de Roble y Raulí}

De acuerdo con Araya y Oyarzun (2000) las formaciones con participación de roble y raulí son las principales exponentes de los bosques de Nothofagus deciduos de hoja grande, los que se encuentran en la sección norte de los bosques templados sudamericanos. En total ocupan actualmente una superficie de 1.682 .685 ha de las cuales en 279.744 ha se encuentra roble y raulí, y en 170.638 ha sólo raulí. Las 1.232 .303 ha restantes corresponden a bosques con presencia de roble, en donde raulí está ausente. Esta superficie de bosque se concentra entre las regiones de Biobío y Los Lagos, teniendo muy poca participación en las regiones de Valparaíso y del Libertador Bernardo O’Higgins.

De acuerdo a Ormazabal y Benoit (1987), la cobertura del roble en el SNASPE 
es baja por cuanto representa un valor muy inferior al 5,8\%, resultante de cotejar sólo a las áreas protegidas con las especie en la región del Maule y la de La Araucanía, correspondientes a 330.000 ha. El raulí está presente en el SNASPE sólo en su rango medio de distribución, es decir, en 8 unidades de la Cordillera de los Andes y una de la Cordillera de la Costa (sector Norte) de la región de La Araucanía, faltando por representar los sectores norte y sur de su distribución (Ormazabal y Benoit, 1987).

\section{Ejemplo de MPBS para Chile}

El modelo propuesto privilegia la conservación in situ en condiciones normales, las cuales deben ser complementadas con métodos de conservación ex situ, en una colaboración público - privada.

Los patrones de variación genética determinan distintos grados de adaptabilidad, tanto entre especies como dentro de ellas, en diferentes áreas de su rango de su distribución biogeográfica. Por esto, las actividades de conservación de genes pueden ser diferentes cuando la especie presenta una extensa distribución, formando grandes poblaciones de cruzamiento aleatorio, o cuando la especie es rara y conforma pequeñas poblaciones más o menos adaptada localmente y con un intercambio limitado de genes. En este sentido, para una especie como el roble, que conforma grandes poblaciones y se distribuye en una amplia variedad de sitios, parece lógico que los métodos de conservación in situ sean los más apropiados y pueden constituir el elemento central del Programa Nacional de Conservación de los Recursos Genéticos de Roble en Chile. En este sentido, las unidades del SNASPE podrían ser la base para una red de subpoblaciones de roble y raulí, manejadas de tal forma de conformar un sistema de conservación de genes dinámico bajo el concepto del MPBS.

Estas áreas de conservación in situ, manejadas, también pueden estar en bosques naturales privados fuera del SNASPE, para lo cual se debería buscar mecanismos de estímulo para que sus propietarios las mantengan.

Como una forma de reducir costos y aumentar la eficiencia del esfuerzo en conservación, se puede considerar unidades in situ conjuntas de roble y raulí cuando estos se encuentren en rodales mixtos, este criterio es válido para muchas otras especies.

Independientemente de lo expuesto para las unidades in situ, la conservación ex situ de recursos forestales, en la forma de ensayos de progenies, es el método más activo y efectivo de conservación (Eriksson et al., 1993). Por esta razón, y dadas las limitaciones de conservar algunas áreas no representadas, ya sea por destrucción de su hábitat natural por desastres o por avance de la frontera agrícola - ganadera, las especies de roble y raulí están representadas inicialmente en dos pares de ensayos de progenie y procedencias, en la zona sur, lo cual puede ser considerado como insuficiente.

$\mathrm{Si}$, por ejemplo, existe interés económico en aprovechar el raulí, los propietarios pueden demandar un programa de mejoramiento genético, donde se armonice directamente la conservación con la producción de semilla. A este respecto existen ejemplos notables, como el huerto semillero clonal de raulí "Huillilemu", iniciado por la Corporación Nacional 
Forestal en 1983, bajo la asesoría de la Cooperativa de Mejoramiento Genético (UACH/ CONAF/Empresas Forestales), el cual fue concebido como un banco para respaldar genotipos selectos y establecido con un diseño que permite utilizarlo como fuente de semillas.

Acciones como la descrita disminuyen la vulnerabilidad del recurso, por cuanto realizan rescate genético de aquellos genotipos que son precisamente los que más se han extraído de los bosques, debido a sus apreciadas características madereras. Aún así, la conservación ex situ de una especie, basada en bancos clonales y huertos semilleros, es estática y no promueve su adaptación en el largo plazo. Sin embargo, es una estrategia útil para especies raras, escasas o con un vulnerable estado de conservación, como el keule, el pitao el ruil y otras, para las cuales los bancos ex situ, adecuadamente aleatorizados, pueden constituir un eficiente instrumento para conservar y acrecentar su variabilidad genética. Para el raulí, los bancos clonales y los huertos semilleros clonales pueden ser considerados como un medio temporal de conservación, a la espera que puedan generar progenies, que finalmente pueden convertirse en la verdadera población de conservación ex situ.

El almacenamiento de largo plazo de semillas, como el efectuado en el banco base de INIA en Vicuña, también representa una instancia de conservación ex situ para aumentar la seguridad de los programas de conservación de genes. Al respecto, una colección de semilla de roble y raulí, representativa de toda el área de distribución biogeográfica de estas especies, ha sido realizada por INFOR y se encuentra respaldada en dicho banco base. No obstante, este procedimiento de conservación ex situ también es estático y debe considerarse sólo como un complemento dentro de la estrategia general de conservación.

Teniendo en cuenta todas las consideraciones anteriores, es posible construir una matriz de prioridades para las especies forestales y arbustivas del bosque nativo chileno. La matriz debe considerar el corto, mediano y largo plazo, e incluir en el muestreo y colecta los bordes de las áreas de distribución de las especies a conservar, por cuanto corresponden a las áreas de mayor variabilidad.

\section{Asignación y Estructuración del Material Genético}

Para una mejor comprensión y optimización del proceso de asignación de material genético a las subpoblaciones, o sublíneas en el sentido de Burdon y Namkoong (1983), es posible apoyarse en la simulación estocástica (POPSIM), propuesta por Mullin y Park (1995).

La conformación de sublíneas permite asignar los recursos genéticos mediante el respectivo valor genético del árbol, o siguiendo patrones geográficos de variación (clinal o ecotípica). En el Programa Nacional se puede integrar el mejoramiento y la conservación (Burdon y Namkoong, 1983) conformando una sola población de mejoramiento, subdividida en sublíneas, pero que cumplirá dos objetivos, mejora y conservación. Por otro lado, las sublíneas o subpoblaciones corresponderán a las poblaciones de procedencias (Vergara, 2000; Ipinza y Gutiérrez, 2000), lo que permitirá aprovechar y proteger su adaptabilidad 
evolutiva, aunque sin menoscabo de que en el objetivo de mejoramiento se privilegien las procedencias más productivas.

Un aspecto administrativo, que tendrá que discutirse al interior de la Red Nacional de Conservación de Recursos Genéticos Forestales, es la normativa y los protocolos para el intercambio de información y material genético, por cuanto su implementación necesariamente deberá considerar el trabajo conjunto de varias instituciones, como la opción más adecuada para enfrentar un programa de esta envergadura.

En la Figura $\mathrm{N}^{\circ} 7$ se muestran las regiones de procedencias bosquejadas por Vergara (2000). En ella se han agregado óvalos traslapados, dispuestos en la gradiente natural de sequía de sur a norte del país, que indican las áreas de muestreo para establecer el modelo de conservación ex situ, bajo el sistema de poblaciones múltiples (MPBS). La región de procedencia es, para una especie o subespecie determinada, la zona o el grupo de zonas sujetas a condiciones ecológicas suficientemente uniformes en las que se encuentran fuentes semilleras o rodales que presentan características fenotípicas o genéticas semejantes, teniendo en cuenta límites de altitud, cuando proceda. Es la unidad básica de comercialización de plantas y semillas forestales, y es el principal medio de establecer una zonificación de las especies con vistas a su conservación. 


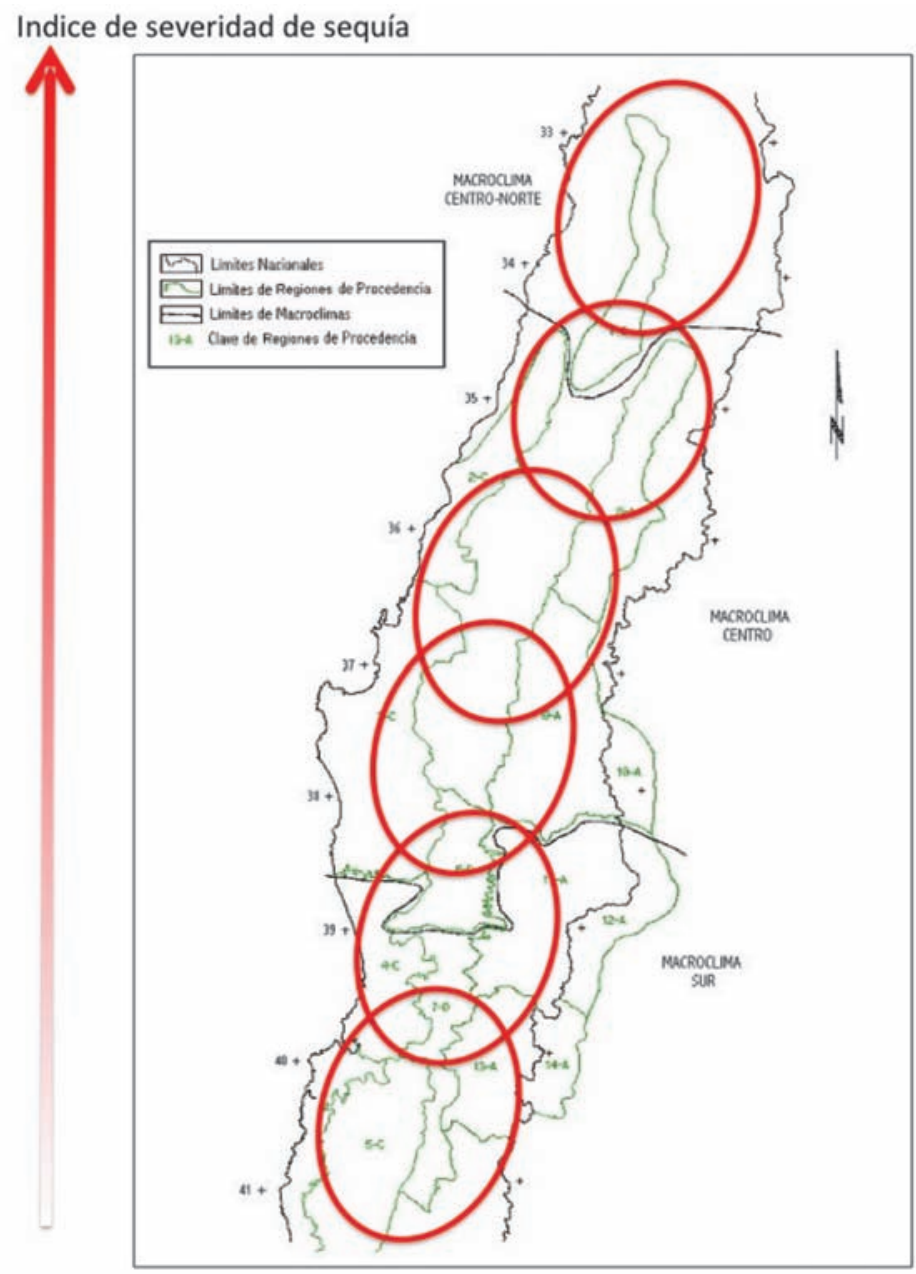

Figura $\mathrm{N}^{\circ} 7$

\section{DISTRIBUCIÓN DE LA SUBPOBLACIONES DE ROBLE Y RAULÍ EN EL MODELO MPBS PARA COLECTAR MATERIAL GENÉTICO PARA LA CONSERVACIÓN EX SITU EN LA GRADIENTE DE SEQUÍA DE SUR A NORTE.}

Con el transcurso del tiempo, y en la medida que el modelo se integre en un programa nacional, donde participen instituciones de distintas regiones, tenderá a asemejarse al sistema de poblaciones múltiples. En tal escenario, las instituciones de cada región podrán orientar las sublineas hacia los caracteres que le sean de interés. Por lo mismo, las poblaciones, hoy sublíneas, tenderán a diferenciarse en un proceso gradual acentuado por las presiones de selección provocadas por el cambio climático que afectará en forma diferencial a cada línea y cada región. 


\section{Redes y Colaboración Internacional}

La conservación de recursos genéticos normalmente trasciende a los límites geográficos de un país, necesitándose actividades cooperativas y de colaboración entre distintos países y organizaciones. En este sentido, el Instituto Forestal de Chile es parte de la Red Latinoamérica de Recursos Genéticos Forestales, LAFORGEN para América Latina y el Caribe, dependiente de Bioversity International (ex - IPGRI), de la cual también depende el programa europeo EUFORGEN de conservación de recursos genéticos. Si bien, en el caso nacional, el alto grado de endemismo de los recursos forestales determina que sus distribuciones naturales y por ende su variabilidad, se concentra al interior del país, es igualmente importante pertenecer a estos programas internacionales de conservación por cuanto, además de la colaboración, la formación y la innovación tecnológica, constituyen un soporte fundamental para la coordinación y el cumplimiento de compromisos globales de conservación.

Además, considerando la relevancia y el reconocimiento de trabajar en red, es recomendable operativizar la creación de una Red Nacional de Recursos Genéticos Forestales, para coordinar la colaboración entre las Universidades, Centros Tecnológicos, el sector privado y en general de todos los actores relacionados con la conservación y utilización de los RGF del país.

\section{CONCLUSIONES}

De acuerdo con los antecedentes presentados, un programa nacional de conservación y mejoramiento de recursos genéticos forestales puede implementarse de acuerdo a las directrices del sistema de mejoramiento por poblaciones múltiples.

Tal programa debería considerar como meta definir especies prioritarias y completar para cada una de ellas, una población principal de 1.000 selecciones, divididas en 20 sublineas con 50 selecciones cada una. Posteriormente, con el objeto de realizar mejoramiento genético se podrá conformar una población élite, donde se incluirían las mejores 40 selecciones evaluadas en plantaciones operacionales o ensayos disponibles. Esta última población es dinámica y transitoria, y sería sometida a un intenso desarrollo para maximizar las ganancias genéticas.

La propuesta presentada cumple con salvaguardar la variabilidad genética de las especies consideradas, asegurando la conservación de su potencial de adaptación ante las modificaciones ambientales, entre ellas las impuestas por el cambio climático, y constituye a su vez una base adecuada para desarrollar programas de mejoramiento genético tendientes al aprovechamiento económico y uso sustentable de los recursos genéticos forestales del país.

La implementación de una estrategia como la propuesta demandará la participación de distintas entidades vinculadas a la conservación y uso sustentable de los RGF, así como la definición de una instancia coordinadora que permita una mayor eficiencia de los esfuerzos y recursos comprometidos en su implementación. 


\section{RECONOCIMIENTOS}

Este trabajo es parte del desarrollo metodológico sobre conservación de Recursos Genéticos Forestales, iniciado el año 2007 en el CIFOR del INIA de Madrid, España, bajo la dirección del Dr. Ricardo Alía.

Los autores agradecen al Dr. Carlos Magni por sus sugerencias en el ámbito de genética poblacional y también al Sr. Santiago Barros por su contribución en la edición de este articulo.

\section{REFERENCIAS}

Araya, L. y Oyarzún, V., 2000. En: Ipinza, R., Gutiérrez, B. y Emhart, V. editores. Domesticación y Mejora Genética de raulí y roble. Universidad Austral / Instituto Forestal. pp. 25-42.

Boshier, David y Michael Henson, 1997. Variación genética. En Cordia alliodora. Genética y mejoramiento de árboles, eds. David Boshier y A. T. Lamb, 43-70. Oxford: Oxford Forestry Institute.

Burdon, R. and Namkoong, G., 1983. Short Note: Multiple Population and Sublines. Silvae Genetica, 32, 5-6

CONAF, CONAMA, BIRF, 1999. Catastro y Evaluación de Recursos Vegetacionales Nativos de Chile. Informe Nacional con Variables Ambientales. 87 p.

Cornelius, Jonathan, Francisco Mesén, E. Correa y Michael Henson, 1996. Variation in Growth and Form of Alnus acuminata Kunth in Costa Rica. Silvae Genetica 45: 24-30.

Danell, O., 1993. Tree breeding strategy: are we too concerned conservationists but inefficient breeders? In: Proceedings of the Nordic group of tree breeding, October 1993, Edinburgh, Scotland. Forestry Commission. pp: 80-94.

EMBRAPA, 2006. El estado del arte de los recursos genéticos en las Américas: conservación, caracterización y utilización. Brasilia, DF, Brasil. 61 p.

Eriksson, G., G. Namkoong and J. H. Roberds, 1993. Dynamic gene conservation for uncertain futures. Forest Ecology and Management 62:15-37.

Eriksson, G., 2000. Red Europea de Conservación de Recursos Genéticos de Frondosas Nobles. Invest. Agr.: Sist. Recur. For.: Fuera de Serie no 2-2000. p. 59-69.

Eriksson, G., Ekberg, I. y Clapham, D., 2006. An introduction to Forest Genetics. Second edition. Genetic Center. Department of Plant Biology and Forest Genetics, SLU. Uppsala. $188 \mathrm{p}$.

Ferreira, M. y Eriksson G., 2006. A Programme for the Management of Forest Tree Genetic Resources in the Azores Islands. Silva Lusitana 14 (1): 59-73. 
Gallo, L., Pastorino, M. y Donoso, C., 2004. Variación en Austrocedrus chilensis (D. Don) Pic. Ser et Bizzarri (Ciprés de la Cordillera). En: Donoso, C., Premoli, A., Gallo, L. y Ipinza, R. Variación Intraespecífica en las especies arbóreas de los bosques templados de Chile. pp. 233- 251.

Gregorius, H.- R., 1980. The probability of losing an allele when diploid genotypes are sampled. Biometrics 36:643-652.

Hubert , J. y Cottrell, J., 2007. The role of Forest Genetic Resources in Helping British Forests Respond to Climate Change. Information Note, Forestry Commision Uk. 12 pp.

INIA-FAO, 2008. Segundo Informe país sobre el estado de los recursos fitogenéticos para la alimentación y la agricultura. Chile 2008. Santiago, Chile. 74

Ipinza, R., 2000. Modelo Básico de Mejora Genética. En: Ipinza, R., Gutiérrez, B. y Emhart, V. editores. Domesticación y Mejora Genética de raulí y roble. Universidad Austral / Instituto Forestal. pp. 197-213.

Ipinza, R. y Gutiérrez, B., 2000. Estrategia de Mejora Genética para Nothofagus alpina y N. obliqua en Chile. En: Ipinza, R., Gutiérrez, B. y Emhart, V. editores. Domesticación y Mejora Genética de raulí y roble. Universidad Austral / Instituto Forestal. pp. 404-417.

Koskela, J., Buck, A. and Teissier du Cros, E., editors, 2007. Climate change and forest genetic diversity: Implications for sustainable forest management in Europe. Bioversity International, Rome, Italy. $111 \mathrm{pp}$.

Kremer, A., 2007. How well can existing forests withstand climate change? In: Koskela, J., Buck, A. and Teissier du Cros, E., editors. Climate change and forest genetic diversity: Implications for sustainable forest management in Europe. Bioversity International, Rome, Italy. pp. 3-17.

LCSAR - MINAGRI - FIA, 2011. Sistema de Innovación de la Agricultura Chilena: Un Plan de Acción hacia el 2030. 68 p.

Lefèvre, F., 2007. Conservation of forest genetic resources under climate change: the case of France. In: Koskela, J., Buck, A. and Teissier du Cros, E., editors. Climate change and forest genetic diversity: Implications for sustainable forest management in Europe. Bioversity International, Rome, Italy. pp. 95-101

Mátyás, C., 2007. What do eld trials tell about the future use of forest reproductive material? In: Koskela, J., Buck, A. and Teissier du Cros, E., editors. Climate change and forest genetic diversity: Implications for sustainable forest management in Europe. Bioversity International, Rome, Italy. pp. 53-69.

Müller - Using, S. y Martin, M., 2011. Manejo de los bosques para la mitigación del efecto invernadero. En: Ipinza, R y Barros, S. editors. El Cambio Climático los Bosques y la 
Silvicultura. Instituto Forestal - MINAGRI. pp. 75-78.

Mullin, T. J., and Park, Y. S., 1995. Stochastic simulation of forest tree breeding populations: a user guide for POPSIM version 2.0.

Navarro, Carlos, Ward, Sheila y Hernández, Marvin., 2002. The Tree Cedrela odorata (Meliaceae): a Morphologically Subdivided Species in Costa Rica. Revista de Biologia Tropical 50: 21-29

Namkoong, G., 1976. A multiple index selection strategy. Silvae Genet. 25:199-201.

Namkoong, G., 1984. A control concept of gene conservation. Silvae Genet. 33:160163.

OCDE, 2005. Evaluaciones del Desempeño Ambiental. Edición en español, Naciones Unidas, CEPAL 246 pp.

OCDE, 2011. Mejores políticas para el desarrollo: Perspectivas OCDE sobre Chile, OECD Publishing. 269 pp.

Ormazabal, C. y Benoit, I., 1987. El Estado de Conservación del Genéro Nothofagus en Chile. Bosque: 8(2): 109-120.

Parques para Chile, 2011. Las áreas protegidas privadas en Chile.

En: http://www.Parquesparachile.cl/index.php?option=com_content\&task=view\&id=89\&lt emid=117. (Consulta agosto, 2011).

Pliûra, A. and. Eriksson, G., 1997. Sustainable gene conservation of Pinus sylvestris in Lithuania. Baltic Forestry 3(1):2-9.

Pliûra, A., 1999. European long-term gene conservation strategies Ash (Fraxinus spp.). En: Turok, J., J. Jensen, Ch. Palmberg-Lerche, M. Rusanen, K. Russell, S. de Vries and E. Lipman, compilers. 1999. Noble Hardwoods Network. Report of the third meeting, 1316 June 1998, Sagadi, Estonia. International Plant Genetic Resources Institute, Rome. pp 12-25.

Pliûra, A., 2004. Possibilities for adaptation of Alnus glutinosa L. to changing environment. BIOLOGIJA. 2004. Nr. 1. P. 6-12.

Salazar, E., León, P., Rosas, M. y Muñoz, C., 2006. Estado de la conservación ex-situ de los recursos fitogenéticos cultivados y silvestres en Chile. Instituto de Investigaciones Agropecuarias. Boletín INIA N 156. Santiago, Chile. 180 p.

Varela, M. and Eriksson, G., 1995. Multipurpose gene conservation in Quercus suber a Portuguese example. Silvae Genet. 44:27-37.

Vergara, R., 2000. Regiones de Procedencia de Nothofagus alpina y N. obliqua. En: Ipinza, R., Gutiérrez, B. y Emhart, V. editores. Domesticación y Mejora Genética de raulí y roble. Universidad Austral / Instituto Forestal. pp. 121-132. 
